\title{
Effect of varying the time frame for COOP-WONCA functional health status charts: a nested randomised controlled trial in Bristol, UK
}

\author{
Tim J Peters, Joanna Coast, Suzanne H Richards, David J Gunnell
}

\begin{abstract}
Study objective-To investigate whether changing the stated time frame for COOP-WONCA charts has any effect on responses. Specifically, to assess the effect of attempting to avoid the situation where the time frame crosses the onset of an acute episode.

Design-A randomised controlled trial of two time frames, nested within a main trial comparing early discharge with a hospital at home scheme against routine discharge policy. The time frames compared were the standard two weeks (four for the pain chart) and a shorter period of 48 hours for all seven charts.
\end{abstract}

Setting-Acute hospital wards in Frenchay Healthcare Trust and the Avon Orthopaedic Centre in Bristol.

Participants-Patients entered into the main trial, who were medically stable, in need of continued rehabilitative care but suitable for discharge to hospital at home. Main results-A total of 200 patients were randomised, 106 to the shorter time frame, 94 to the standard charts. No clear differences were observed between the two groups for the proportion failing to self complete the charts. For the (seven) chart scores, only pain was statistically significantly different between the time frames (Mann-Whitney $p=0.0085$; proportion reporting moderate or severe pain $19 \%$ higher in the standard group, $\mathbf{9 5 \%}$ confidence intervals $5 \%$ to $33 \%$ ). For both this chart and that for change in health, however, there was evidence of greater differences between the versions of the chart among those admitted more recently ( $p$ values for relevant interactions 0.004 and $<0.001$ respectively).

Conclusions-While the present findings give some support for the wide applicability of the standard version, there is sufficient evidence here to indicate that the time frame may influence the results, particularly for patients with a recent acute episode. In the absence of further data, then, it would seem prudent to consider a shorter time frame for such patients, especially if the aim is to assess current health status or to measure changes over a comparatively short period of time, or both.

(F Epidemiol Community Health 1998;52:59-64)
The COOP-WONCA charts aim to provide measures of functional health status. ${ }^{12}$ There are seven charts, each using words and pictures to convey five (ordered) levels of functional health status. They represent: physical fitness, feelings, daily activities, social activities, change in health, overall health, and pain (the latter being optional). For the first six the standard time frame is the previous two weeks, but for the pain chart it is the previous four weeks. The wording in the stem of each chart is as follows, using overall health as an example: "During the past 2 weeks... How would you rate your health in general?" 2

The acceptability, reliability, and validity of the charts have been assessed both within the $\mathrm{UK}^{3}$ and elsewhere. ${ }^{1245}$ Although the charts have mainly been validated for use in primary care, ${ }^{35-7}$ a wide variety of acute and chronic conditions have been covered, and evaluations have taken place in various settings including diabetes and hypertension clinics. ${ }^{8}$ In addition, a population based sample has been studied to demonstrate the value of the charts for epidemiological purposes. ${ }^{9}$ Nevertheless, it is within the context of comparatively chronic conditions that the time frame of the last two weeks is likely to be most consistent with the stated aim of measuring current health status.

In this study, the COOP-WONCA charts were being used as part of a randomised controlled trial comparing early discharge with a hospital at home scheme with continued acute hospital care for a mainly elderly patient group.(Coast J et al, unpublished report to the South \& West NHS R\&D Directorate). The charts were part of a battery of outcome measures, and were chosen over other health status measures (such as the SF-36 ${ }^{10}$ ) on account of their simplicity and feasibility for use with a predominantly frail elderly group of patients. Indeed, these charts have been found to be acceptable to, and easily completed by, patients experiencing an acute episode. ${ }^{57}$ In addition, it is possible for them to be either self completed or interviewer administered. ${ }^{8}$

The time frame traditionally covered by such measures of functional health status has been selected so that it is long enough to avoid excessive short-term influences, and short enough to be sensitive to changes. For the acute hospital patients in this study, however, it was anticipated that the standard two week time frame might cross both the onset of the acute episode and the subsequent admission to hospital. That is, the time frame for what is intended to be "current" health status might 
encompass two very different health statesthose before and after the onset of the acute episode. $^{57}$ The opportunity was therefore taken to assess the relative impact of using a 48 hour time frame rather than the longer, conventional period of two weeks (four weeks for the pain chart) by conducting a nested randomised controlled trial of time frame within the context of the main trial.

Specifically, the aim of this nested trial was to investigate whether changing the stated time frame on the COOP-WONCA charts had any effect on responses among this group of elderly hospital patients. This was tested by comparing responses when they were completed at the baseline assessment for the main trial, according to whether the standard (two/four week) or a shorter (48 hour) time frame was stated on the charts. The question of the suitable time frame has been raised previously, particularly for acute patients, with suggestions of a much shorter time frame for at least some of the charts. $^{57}$ There is, however, no previous attempt to consider this question formally.

\section{Methods}

Briefly, the main trial comprised 241 patients recruited over 16 months from July 1994 to October 1995 from the Frenchay Healthcare Trust and the Avon Orthopaedic Centre in Bristol. Approval from the relevant ethics committees was obtained. Patients who were considered by the clinical team to be appropriate for hospital at home care (that is, medically stable but in need of continued rehabilitation/ nursing care and with appropriate home circumstances) and who also consented to participate in the study, were randomised on a $2: 1$ basis to either early discharge to a hospital at home scheme or continued acute hospital care with routine discharge. Of the 241 patients, most $(64 \%)$ were recruited from the orthopaedic specialty, with the remainder coming from care of the elderly (29\%), general medicine, general surgery, and neurology. (Coast J et al, unpublished report to the South \& West NHS R\&D Directorate).

Of this total, the first 30 patients were not included in the trial reported here, because the design catering for the present research question was only fully developed after they had already been entered into the trial. A further 11 patients were admitted to the main trial, but because of extreme confusion/dementia were not requested to complete any of the patient questionnaires. The remaining 200 patients were randomised to two different versions (time frame either 48 hours or the standard of two/four weeks) of the COOP-WONCA charts to be completed at the time of recruitment to the main trial. Randomisation was stratified across the two arms of the main trial and hence is nested within it; in other words, randomisation to the two time frames was carried out separately for the "hospital at home" and "hospital" arms of the main trial. It was performed independently of the staff recruiting patients into the trial, and at recruitment all staff and potential participants were unaware of the allocations for either the main or the nested trial.
KEY POINTS

- The standard versions of the COOPWONCA functional health status charts are reasonably widely applicable.

- The time frame adopted by functional health status measures can influence the results.

- This effect seems to be exaggerated among patients with a more recent acute episode of ill health.

- It is both feasible and worthwhile to nest randomised evaluations of methodological issues within wider main studies.

Using the example of the physical fitness chart, the wording of the baseline COOPWONCA charts was changed for a random half of the patients, from the standard: During the past 2 weeks... What was the hardest physical activity you could do for at least 2 minutes? to: During the past 48 hours... What was the hardest physical activity you could do for at least 2 minutes?

In no other respect was the wording of either the questions or the response categories in the charts changed.

The procedures adopted for the main trial were unaffected by the randomisation to COOP-WONCA chart time frame, and indeed the latter was only carried out for the baseline assessments - all charts applied at the follow up studies used the standard time frames. The charts were intended to be self completed in this study wherever possible, but because of the frail, elderly nature of many of the patients the option of interviewer administration had to be made available.

Although the sample size was principally determined by the criterion of the main trial, (Coast J et al, unpublished report to the South \& West NHS R\&D Directorate) the 200 patients available for the nested (time frame) randomisation yielded $80 \%$ power to detect differences of approximately 0.4 standard deviations at the two sided $5 \%$ significance level.

\section{STATISTICAL METHODS}

Baseline comparability of the two groups in the nested trial was assessed using descriptive statistics of demographic characteristics and measures of activities of daily living (the Barthel Index) ${ }^{11}$ and cognitive functioning (the Folstein score). ${ }^{12}$ Similarly, descriptive statistics by group were obtained for the time between admission to hospital and completion of the charts, and for whether or not the charts were self completed. Both these variables were regarded as a priori stratifying factors.

Failure to self complete the COOPWONCA charts was also considered as an outcome variable in its own right, as differences between the chart versions could reflect differential confusion caused by wording. For this purpose, the proportions failing to self complete were compared between the groups using a $\chi^{2}$ test and associated $95 \%$ confidence intervals. The key comparisons between the two 
Table 1 The cut points used for the COOP-WONCA charts in order to delineate a relatively "poor" outcome

\begin{tabular}{lll}
\hline & \multicolumn{2}{c}{ Definition of "poor" outcome } \\
\cline { 2 - 3 } Chart & Categories & Labels \\
\hline Physical fitness & 5 & "Very light, (for example) walk at a slow pace or not able to walk at all" \\
Feelings & 3,4, and 5 & "Moderately", "quite a bit", and "extremely" bothered by emotional problems \\
Daily activities & 4 and 5 & "Much difficulty" and "could not do" \\
Social activities & 3,4 , and 5 & "Moderately", "quite a bit", and "extremely" limited \\
Change in health & 3,4, and 5 & "About the same", "a little worse", and "much worse" \\
Overall health & 4 and 5 & "Fair" and "poor" \\
Pain & 4 and 5 & "Moderate" and "severe" \\
\hline
\end{tabular}

time frames, though, were of the scores on the COOP-WONCA charts themselves. Given the ordinal nature of these charts, and the highly skewed distributions of many of them for this group of patients, these comparisons were performed using Mann-Whitney $\mathrm{U}$ tests. ${ }^{2}$ These constitute the primary analysis for each chart and use the original scores, thereby avoiding for these analyses any need to dichotomise the chart scales.

To provide some indication, though, of the possible magnitude of the differences between the groups (and also to facilitate adjustment using logistic regression), the proportions of scores above given cut off points were calculated, together with $95 \%$ confidence intervals for differences between them. As higher scores on the charts represent worse health status, these proportions represent the relative frequency of "poor" outcome. These cut off points (detailed in table 1) were determined for each chart before inspection of the differences in distributions of the scores across the groups; they were selected by a combination of a priori grounds bearing in mind the type of participants in the trial and the observed distributions of the charts for all patients combined.

Secondary (stratified) analyses investigated whether the comparisons of the outcome measures were accounted for by any notable baseline differences in patient characteristics between the groups. In addition, as the primary hypothesis of the nested trial was developed because of concerns that the two week time frame could cross the onset of the acute patient episode, further sub-group analyses were carried out stratifying by whether or not the date of admission was within the two weeks before the date of completion of the COOP-WONCA charts (four weeks for the pain chart). Given that this stratification was a prior hypothesis, this was subjected to detailed analysis. In particular, as well as simple $\chi^{2}$ analyses for the

Table 2 Baseline characteristics of patients allocated to the 48 hour and standard time frames on the COOP-WONCA charts

\begin{tabular}{lll}
\hline & $\begin{array}{l}\text { 48 hour time frame }(n=106) \\
\text { frequency }(\%)\end{array}$ & $\begin{array}{l}\text { Standard time frame }(n=94) \\
\text { frequency }(\%)\end{array}$ \\
\hline Age (y) & $29(27)$ & \\
$<75$ & $58(55)$ & $37(39)$ \\
$75-84$ & $19(18)$ & $20(21)$ \\
$85+$ & $76(72)$ & $64(68)$ \\
Sex (female) & $37(36) \dagger$ & $33(35)$ \\
Social class (manual) & $70(66)$ & $63(67)$ \\
Specialty (orthopaedics) & $16(14,17) \dagger$ & $16(15,17) \ddagger$ \\
Barthel score & $26(23,27) \dagger$ & $26(24,28)$ \\
\hline Folstein score & & \\
\hline
\end{tabular}

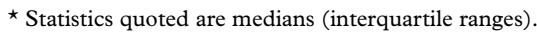

† Two patients had missing data.

$\ddagger$ One patient had missing data. two strata separately, the interactions between COOP-WONCA chart version and the time from admission to completion were tested for the proportions scoring above each of the chart cut off points using logistic regression models. In these models, the (binary) outcome was the proportion scoring above the cut off point for the relevant chart; the explanatory variables were the chart version (that is, randomisation group) plus time from admission to completion of the charts. The same procedure was adopted for stratification by ability to self complete the charts.

All statistical analyses were carried out on an intention to treat basis, using a two sided 5\% significance level. As with all analyses of the COOP-WONCA charts, there is a danger of detecting differences that are purely a result of chance, because of the multiple significance testing involved. Care has been taken here, however, to identify a relatively small number of issues to investigate, in particular for the (primary) stratified analyses. Notwithstanding this, the Bonferroni correction was considered at appropriate points in the analyses; in general, given that there are seven charts, for a $5 \%$ experiment wise significance level, tests for individual charts would under this correction only be regarded as significant if the $\mathrm{p}$ value fell below 0.0071. The statistical packages used were SAS, CIA, and EGRET.

\section{Results}

PATIENT CHARACTERISTICS AND STRATIFICATION VARIABLES

The age range of the 200 patients $(70 \%$ of whom were female) in the nested trial was 46 to 92 years, with a median of 79 and an interquartile range of 72 to 84 years. Sixty (30\%) were fractured neck of femur patients, $42(21 \%)$ patients had had various other fractures, 39 (20\%) were receiving elective hip or knee replacements, $18(9 \%)$ had had strokes, and the remaining $41(20 \%)$ had experienced miscellaneous conditions.

Table 2 shows the characteristics of patients in each of the two groups separately, 106 being randomised to receive the 48 hour time frame and the remaining 94 the standard charts. The only potentially influential difference is that, although the median age is 79 years in both groups, the spread of ages is greater in the standard chart group, particularly at the lower end (table 2). In all the other respects considered, the two groups were very similar-in particular, there were no differences with respect to either activities of daily 
Table 3 Descriptive statistics of a priori stratification variables in the two COOP-WONCA time frame groups

\begin{tabular}{lll}
\hline & $\begin{array}{l}\text { 48 hour time frame } \\
(n=106) \text { frequency (\%) }\end{array}$ & $\begin{array}{l}\text { Standard time frame } \\
(n=94) \text { frequency (\%) }\end{array}$ \\
\hline Admission within 2 wk prior to completion & $51(50)^{\star}$ & $46(49) \ddagger$ \\
Admission within 4 wk prior to completion & $75(74)^{\star}$ & $70(75) \ddagger$ \\
Failure to self complete the charts & $52(51) \dagger$ & $40(43) \ddagger$ \\
\hline
\end{tabular}

* Five patients had missing data

† Four patients had missing data.

$\ddagger$ One patient had missing data.

living or cognitive functioning, each of which arguably provides stronger evidence of comparability than age.

Table 3 presents the descriptive statistics for the a priori stratification variables. There are no differences for either of the relevant cut off points of time from hospital admission to completion of the charts, but there is a potentially influential difference of $8 \%$ in the proportions failing to self complete the charts (table 3 ).

FAILURE TO SELF COMPLETE THE COOP-WONCA CHARTS

In its role as an outcome variable, the difference of $8.0 \%$ between the proportions failing to self complete the charts (table 3 ) is not statistically significant $(95 \%$ confidence intervals -6.0 to $+21.9 \% ; p=0.26)$. Stratification across the three age groups in table 2 did not change this result (data not shown); although the difference was reduced in the middle age group, the small sample sizes and secondary nature of this analysis mean that such suggestions should be interpreted with extreme caution.

There was a clear differential effect, though, when stratifying failure to self complete by whether or not the admission took place within two weeks before completion of the charts. Overall, those with more recent admissions were much more likely to have completed the charts themselves (two thirds compared with just less than $40 \%$ for those admitted more than two weeks previously; $\mathrm{p}<0.001$ from logistic regression with or without adjusting for chart time frame). Furthermore, the difference between the two time frames in terms of the proportion failing to self complete was only apparent among those admitted more recently (observed difference of nearly $20 \%$ in this subgroup, with a $\mathrm{p}$ value from $\chi^{2}$ analysis of 0.051 ). The formal test of interaction on the whole data set, however, was not statistically significant $(p=0.15)$, and so chance may well explain this finding.
THE COOP-WONCA CHART SCORES

The total numbers of patients with missing data on individual charts were small: six for each of feelings, social activities, and change in health; seven for physical fitness; eight for overall health; nine for pain, and 16 for daily activities. Table 4 shows the primary outcome analyses involving the comparisons of the various COOP-WONCA chart scores across the two time frame groups. As can be seen, the only comparison to reach statistical significance is that for the pain chart, and even this would be borderline under the Bonferroni correction. Although those for physical fitness and daily activities approach significance, the overall finding is of little difference between the results for the two versions. The analyses of proportions with "poor" outcomes yields the same conclusion in terms of statistical significance, with only the confidence interval for the pain chart not including zero. With respect to direction and magnitude, negative values for the differences in table 4 indicate better health status as measured by the 48 hour version. Although the difference in pain chart scores is clearly in this direction, there is no consistent pattern across the remaining six charts. The confidence intervals for both the pain chart and the other comparisons are quite wide, reflecting the sample sizes and the fact that for their calculation the chart scores have been dichotomised.

The comparisons for the chart scores were then repeated after stratification, first for age group, second for time between admission and completion of the charts (with a two week cut off point for all but the pain chart, for which a four week interval is appropriate), and third for ability to self complete the charts. Stratification by age had almost no effect on the comparisons for the charts across the two groups, though there was a suggestion that the differences for pain were diminished for the middle age group. As with the self completion outcome, though, stratification according to date of admission indicated that for almost all charts (apart from overall health) the differences were exaggerated among the more recent admissions-again an apparent "interaction". That is, the differences between the responses according to time frame were most pronounced among those for whom the standard time frame would be more likely to have included the onset of the acute condition. From formal tests of significance, though, only the interactions for change in health and pain were statistically significant

Table 4 Levels of statistical significance for comparisons of COOP-WONCA chart scores between the two time frames, proportions of chart scores above given cut points, differences between these proportions and their 95\% confidence intervals $(95 \% \mathrm{CI})$

\begin{tabular}{llllll}
\hline Chart & $p$ value & $\begin{array}{l}\text { Proportion (\%) } \\
(48 h)\end{array}$ & $\begin{array}{l}\text { Proportion (\%) } \\
\text { (standard) })\end{array}$ & $\begin{array}{l}\text { Difference in } \\
\text { proportions (\%) } \neq\end{array}$ & $95 \%$ CI for difference (\%) \\
\hline Physical fitness & 0.085 & 78.2 & 68.5 & 9.7 & $(-2.7,+22.2)$ \\
Feelings & 0.92 & 32.4 & 35.9 & -3.5 & $(-16.9,+9.8)$ \\
Daily activities & 0.091 & 62.5 & 51.1 & 11.4 & $(-2.9,+25.6)$ \\
Social activities & 0.35 & 42.2 & 52.2 & -10.0 & $(-24.0,+4.0)$ \\
Change in health & 0.15 & 40.2 & 47.8 & -7.6 & $(-21.6,+6.3)$ \\
Overall health & 0.14 & 49.5 & 39.6 & 9.9 & $(-4.1,+23.9)$ \\
Pain & 0.0085 & 40.0 & 59.3 & -19.3 & $(-33.3,-5.4)$ \\
\hline
\end{tabular}

* From Mann-Whitney U tests.

† The standard time frame is 2 weeks for all charts, except pain where it is 4 weeks.

$\ddagger$ Based on proportion with poor chart scores in 48 hour group minus that in the standard group. 
$(\mathrm{p}<0.001$ and $\mathrm{p}=0.004$ respectively). The other $p$ values were: physical fitness $(p=0.96)$; feelings $(p=0.28)$; daily activities $(p=0.49)$; social activities $(p=0.32)$; and overall health $(p=0.20)$. The nature of these interactions (which would remain significant even after the Bonferroni correction) can be illustrated by the results for the pain chart, as follows.

As measured by the pain chart, the health status of those admitted more recently was on average poorer than that of the other patients ( $p=0.009$ by $\chi^{2}$ analysis of proportions). Moreover, the difference in the proportion reporting moderate or severe pain across the chart time frame groups was not significant for the patients admitted more than four weeks before completion of the charts $\left(p=0.21\right.$ by $\chi^{2}$ analysis). For those admitted more recently, however, the proportion reporting high levels of pain was much higher for the standard (four week) version than the 48 hour version (a difference of $-30 \%$ with a $95 \%$ confidence intervals of $-46 \%$ to $-15 \%$ and a $\mathrm{p}$ value of 0.0003 by $\chi^{2}$ analysis). As noted above, the formal test for this interaction was highly statistically significant $(p=0.004$ from logistic regression). An extremely similar set of results was obtained for the outcome of change in health being either the same or worse over the period of the stated time frame.

The third and final stratification was according to ability to self complete the charts. From logistic regression models, the $\mathrm{p}$ values for the interactions between time frame and ability to self complete were: physical fitness $(p=0.11)$; feelings $(p=0.69)$; daily activities $(p=0.77)$ social activities $(p=0.66)$; change in health $(\mathrm{p}=0.025)$; overall health $(\mathrm{p}=0.51)$; and pain $(p=0.19)$. The only significant differential effect, then, was for change in health, where the difference between the time frames was larger among patients who completed the charts themselves. However, this was only marginally significant before correction for multiple testing, and not significant after applying the Bonferroni correction. There were no other effects of this stratification-for instance, controlling for ability to self complete had no impact either on the interactions described above between date of admission and time frame or on the overall effect of time frame on pain chart scores.

\section{Discussion}

Regarding the primary outcomes of the chart scores, there were few consistent differences between the results obtained with the two versions of the COOP-WONCA charts. Only one was statistically significant (pain), and this is the sole chart of the seven that is held to be optional-it has indeed often not been used. ${ }^{359}$ It is also the only chart with the longer (four week) time frame. The confidence intervals for the differences in proportions are quite wide, though, so in this respect the precision of this study does not entirely rule out potentially important differences between the time frames. Only one overall test was significant using the more sensitive Mann-Whitney tests, however, and certainly there was no clear pattern to the direction of possible differences between the time frames across the charts. One potential explanation for this is that patients in acute settings are more influenced by their current functional health status than by the requested time frame.

Notwithstanding the above general findings, for almost all outcomes the differences were exaggerated in the patients admitted to hospital more recently - it being in these patients that the concerns (about the time frame crossing the acute episode) are likely to be most relevant. In only two charts is this differential effect (or interaction) formally statistically significant, though a larger sample size may indicate that this tendency is one that should be borne in mind when applying the charts-both in terms of the scores themselves and the ability of patients to self complete the charts in the first place.

The result that patients admitted more recently were in general much more likely to be able to self complete the charts may be a result of a variety of factors; as might be expected, self completion of other instruments has been found to be related to age (itself related to length of admission) and cognitive ability. (Coast J et al, unpublished report to the South \& West NHS R\&D Directorate). The most probable explanation, however, is simply that those patients who had been in hospital for a longer time before entry into the main trial were those who were relatively unwell in the first place or had experienced complications, or both. Stratification by ability to self complete the charts had in general no effect on the comparisons of the two versions of the charts.

As regards the directions of the significant interactions, the pattern in both cases is that among the recent admissions, the standard version indicates a worse health status than does the shorter time frame ( 48 hour) version. The most probable explanation for this is that encompassing the acute episode (which for many patients was a fracture of one kind or another) yields a poorer assessment of health (specifically, pain and change in health) compared with a time frame that is less likely to include the episode. Moreover it is plausible that a shorter time frame would lead to lower levels of reported pain, because during the whole of this time (in hospital) the patient is much more likely to have had adequate analgesia.

Potential shortcomings of this study include the fact that it took place in a hospital environment, using charts that have largely been validated for use in primary care.$^{3-7}$ No important differences in psychometric performance across various settings were apparent in early developmental work in the US, however, ${ }^{8}$ and the charts have been found useful for acute episodes of a chronic condition, namely asthma. ${ }^{7}$ Notwithstanding the low levels of missing data here (which are certainly no worse than those in other settings ${ }^{39}$ ), there remains a need for fundamental studies of reliability and validity of the charts in a variety of secondary care settings and for comparisons between self completion and interviewer administration. 
The generalisability of the findings of this study to other patient groups also remains to be demonstrated - particularly younger groups of subjects amongst whom charts such as physical fitness may be more sensitive.

Applying the (quite conservative) Bonferroni correction to the test wise level of statistical significance for the seven charts leads to only $p$ values less than 0.0071 as being reliable in this sense. Given the results presented above, and the conservatism of this correction, this issue does not fundamentally change the conclusions to be drawn from this study. In particular, the two interactions still attain statistical significance, and the overall test for pain is borderline.

The lack of precision for the analyses of the dichotomous outcomes is a reflection of the sample size, which was, as stated above, determined by the requirements of the main trial. Nevertheless, this was reasonable in terms of power for quantitative outcomes and, from a wider perspective, the information gleaned in the trial reported here could be contended to have been derived in a highly efficient manner. The marginal resources required to conduct the trial of COOP-WONCA chart time frame were comparatively small (nested as it was within an ongoing trial); moreover, given that only baseline assessments were involved, the potential disruption of the main trial was negligible whatever the findings regarding time frame.

In conclusion, questions regarding the use of the COOP-WONCA charts for patients experiencing an acute episode have been raised previously-specifically, in the contexts of acute episodes of asthma ${ }^{7}$ and low back pain. ${ }^{5}$ No empirical evidence accompanied these concerns, however, and so the present findings should be investigated further. Certainly they need to be replicated in a variety of settings before definitive conclusions can be drawn about the desirability or otherwise of changing the time frame on the COOP-WONCA charts - and particularly the validity of using various specific versions in different contexts, including different patient groups. The same issue arises for any health status measure, particularly generic ones relating to a period of time as in, for example, the SF-36, which specifies the previous four weeks ${ }^{10}$; methodological studies are apparently underway to consider the issue of time frame for the SF-36, particularly for acute conditions. ${ }^{13}$
While the present findings give some support for the wide applicability of the standard version of the COOP-WONCA charts, there is sufficient evidence to show that the time frame may influence the results, particularly for patients with a recent acute episode. In the absence of further data, then, it would seem prudent to consider a shorter time frame for such patients, especially if the aim is to assess current health status or to measure changes over a comparatively short period of time, or both.

We are most grateful to the staff of the Frenchay Healthcare Trust and the Avon Orthopaedic Centre at Southmead HospiTrust and the Avon Orthopaedic Centre at Southmead Hospi-
tal, Bristol for all their help and cooperation in the conduct of tal, Bristol
this study.

Funding: this research was funded by the South \& West NHS Research \& Development Directorate.

Conflicts of interest: none.

1 WONCA Classification Committee, ed. Functional status measurement in primary care. New York: Springer-Verlag, 1990.

2 Scholten JHG, Van Weel C. Functional status assessment in family practice: the Dartmouth COOP functional health assessment charts/WONCA. Lelystad: MEDITekst, 1992.

3 Kinnersley P, Peters T, Stott N. Measuring functional health status in primary care using the COOP-WONCA charts: acceptability, range of scores, construct validity, reliability and sensitivity to change. Br f Gen Pract 1994;44:545-9.

4 Nelson E, Wasson J, Kirk J, et al. Assessment of function in routine clinical practice: description of the COOP chart method and preliminary findings. F Chron Dis 1987;40 (suppl 1):55-63S.

5 Andres E, Temme M, Raderschatt B, Szecsenyi J, Sandholzer H, Kochen MM. COOP-WONCA charts: a suitable functional status screening instrument in acute low back pain? Br F Gen Pract 1995;45:661-4.

6 Meyboom-de Yong B, Smith RJA. Studies with the Dartmouth COOP charts in general practice: comparison with the Nottingham Health Profile and the General Health Questionnaire. In: WONCA Classification Committee, ed. Functional status measurement in primary care.

7 Wong-Chung D, Mateijsen N, West R, Ravell L, Van Weel C. Assessing the functional status during an asthma attack with Dartmouth COOP charts. Validity with respect to the change in asthma. Fam Pract 1991;8:404-8.

8 Nelson EC, Landgraf JM, Hays RD, et al. The COOP function charts: a system to measure patient function in physicians' offices. In: WONCA Classification Committee, ed. Functional status measurement in primary care. New York: Springer-Verlag, 1990:97-131.

9 Bruusgaard D, Nessioy I, Rutle O, Furuseth K, Natvig B. Measuring functional status in a population survey. The Dartmouth COOP functional health assessment charts/ WONCA used in an epidemiological study. Fam Pract 1993;10:212-18.

10 Brazier JE, Harper R, Jones NMB, O'Caithin J, et al. Validating the SF-36 Health Survey Questionnaire. BMf 1992;305:160-4.

11 Mahoney FI, Barthel DW. Functional evaluation: the Barthel Index. Maryland State Med f 1965;14:61-5.

12 Folstein MF, Folstein SE. "Mini-mental state". A practical method for grading the cognitive state of patients for the clinician. I Psychiatr Res 1975;12:189-98.

13 Ware JE. SF-36 Health Survey: manual and interpretation guide. Boston: The Health Institute, New England Medical Center, 1993. 